\title{
A PRIORI ERROR ESTIMATES FOR APPROXIMATION OF PARABOLIC BOUNDARY VALUE PROBLEMS*
}

\author{
R. E. SHOWALTER †
}

\begin{abstract}
The $L^{2}$-error estimates are established for the continuous time Faedo-Galerkin approximation to solutions of a linear parabolic initial boundary value problem that has elliptic part of order $2 m$. Properties of analytic semigroups are used to obtain these estimates directly from the $L^{2}$-estimates for the corresponding steady state elliptic problem under hypotheses only on the data in the problem (initial condition, elliptic operator).
\end{abstract}

1. Introduction. We obtain estimates for the error resulting from a continuous time Faedo-Galerkin approximation of the linear parabolic boundary value problem

$$
u^{\prime}(t)+A u(t)=0, \quad t>0, \quad u(0)=u_{0},
$$

where $A$ is a realization in $L^{2}(G)$ of an elliptic partial differential operator of order $2 m$. These estimates are best possible: the rate of convergence is the same as that for the Galerkin approximation of the corresponding (variational) elliptic steady state problem whose exact solution is the initial condition, $u_{0}$.

These estimates for the rate of convergence are well known; our contribution here is that they are obtained from hypotheses on the data in the problem - the regularity properties of the elliptic operator, an approximation assumption on the rate of convergence in the corresponding elliptic problem and the initial condition - and without the usual ad-hoc assumptions on the solution $u(\cdot)$ of the problem. The proofs depend on the existence-regularity theory for the evolution problem. See [1], [2], [12] for related results.

An exposition of the well-known results for the steady state problem is given in $\S 2$, where we briefly discuss the approximation of solutions and the interpolation of various estimates associated with these regular elliptic boundary value problems. Section 3 begins with a description of the regularity properties of the solution of the abstract Cauchy problem (1.1) where $-A$ is the generator of an analytic semigroup of contractions. We use the notions of interpolation theory and fractional powers of the operator $A$ to relate the growth of $u(t)$ in various norms as $t \rightarrow 0^{+}$to the (regularity of the) initial condition, $u_{0}$. After these preliminaries, the error estimates are proved and stated as our Theorem.

2. Elliptic operators: Interpolation and approximation. Let $V$ and $H$ be Hilbert spaces for which $V$ is a dense subspace of $H$ and the injection is continuous. Denote the inner product and norm on $H$ by $(\cdot, \cdot)$ and $|\cdot|$, respectively, and the norm on $V$ by $\|\cdot\|$. Let $a(\cdot, \cdot)$ be a continuous bilinear form on $V$ which is coercive: there is a

* Received by the editors March 29, 1974, and in revised form August 5, 1974.

† Department of Mathematics, University of Texas, Austin, Texas 78712. This work was supported in part by the National Science Foundation under Grant GP-34261. 
number $c>0$ such that

$$
a(v, v) \geqq c\|v\|^{2}, \quad v \in V
$$

The triple $\{a(\cdot, \cdot), V, H\}$ defines an unbounded operator $A$ on $H$ with domain $D(A)$ by

$$
a(u, v)=(A u, v), \quad u \in D(A), \quad v \in V,
$$

where $D(A)$ is the set of all $u \in V$ for which the linear form $v \mapsto a(u, v)$ is continuous on $V$ with the (weaker) norm of $H$. From (2.1) it follows that $A$ is an injection of $D(A)$ onto $H$, and $D(A)$ with the norm

$$
\|v\|_{A}=|A v|, \quad v \in D(A),
$$

is a Hilbert space (with the obvious inner product). Further, $D(A)$ is dense in $H$, and the identity map from $D(A)$ into $H$ is continuous: there is a number $c_{1}>0$ such that

$$
\|v\|_{A} \geqq c_{1}|v|, \quad v \in D(A) .
$$

Fractional powers of operators such as $A$ have been constructed and discussed by many authors. (See the bibliographies of [3], [6], [10].) For each $\alpha, 0<\alpha<1$, there is an operator $A^{\alpha}$ which is a closed linear injection with dense domain $D\left(A^{\alpha}\right)$ and range $H . D\left(A^{\alpha}\right)$ is a Hilbert space with norm

$$
\|v\|_{A^{\alpha}}=\left|A^{\alpha} v\right|, \quad v \in D\left(A^{\alpha}\right) .
$$

and $A^{\alpha}$ has properties appropriate for the $\alpha$ th root of $A$. Under hypotheses more general than those above on $A, \mathrm{~T}$. Kato proved the following [5].

Let $A_{1}$ be an unbounded operator on a Hilbert space $H_{1}$ with properties like $A$ on $H$. If $T \in L\left(H, H_{1}\right)$ and $T \in L\left(D(A), D\left(A_{1}\right)\right)$, where $L(X, Y)$ denotes the Banach space of continuous linear maps of $X$ into $Y$, then for each $\theta, 0<\theta<1$, we have $T \in L\left(D\left(A^{\theta}\right), D\left(A_{1}^{\theta}\right)\right)$ with the estimate

$$
\|T\|_{L\left(D\left(A^{\theta}\right), D\left(A_{1}^{\theta}\right)\right)} \leqq c_{2}\|T\|_{L\left(H, H_{1}\right)}^{1-\theta}\|T\|_{L\left(D(A), D\left(A_{1}\right)\right)}^{\theta} .
$$

This is an interpolation theorem which we can apply with $T=A_{1}=I$ on $H_{1}$ $=D\left(A_{1}\right)=H$ to obtain from (2.2) the estimate

$$
\left|A^{\theta} v\right| \geqq c_{1}^{\theta}|v|, \quad 0 \leqq \theta \leqq 1, \quad v \in D\left(A^{\theta}\right)
$$

There are various equivalent methods of constructing from a pair of Hilbert spaces $H_{0}$ and $H_{1}$, with $H_{0}$ dense and continuously embedded in $H_{1}$, a family of intermediate spaces $\left[H_{0}, H_{1}\right]_{\theta}, 0<\theta<1$, and we refer to [10] for references. Such spaces have an interpolation property like (2.3) for bounded operators, and we have (e.g., [9])

$$
[D(A), H]_{\theta}=D\left(A^{1-\theta}\right), \quad 0 \leqq \theta \leqq 1,
$$

with equivalent norms, for the operator $A$ on $H$ constructed above.

In order to construct an elliptic differential operator, we let $G$ be a bounded open set in $\mathbb{R}^{n}$ with smooth boundary $\partial G$ locally on one side of $G$, and define for integer $m \geqq 0$ the Hilbert space $H^{m}(G)$ of (equivalence classes of) functions $v \in L^{2}(G)$ 
$\equiv H^{0}(G)$ such that each (distribution) derivative $D^{p} v$ of order $|p| \leqq m$ belongs to $L^{2}(G)$. The inner product on $H^{m}(G)$ is

$$
(u, v)_{m}=\sum\left\{\int_{G} D^{p} u(x) D^{p} v(x) d x:|p| \leqq m\right\}
$$

and the norm is given by $\|v\|_{m}=(v, v)_{m}^{1 / 2}([8])$. Let $V$ be a closed subspace of $H^{m}(G)$ containing the space $C_{0}^{\infty}(G)$ of infinitely differentiable functions with compact support in $G$. Take $H=L^{2}(G)$, so $V$ is dense in $H$, and prescribe a bilinear form on $V$ by

$$
a(u, v)=\sum\left\{\int_{G} a_{p q}(x) D^{q} u(x) D^{p} v(x) d x:|p|,|q| \leqq m\right\},
$$

where the coefficients $a_{p q}$ are smooth functions on $\bar{G}$ and the coercive estimate (2.1) holds. The unbounded operator $A$ determined by the triple $\{a, V, H\}$ is given by

$$
A u=\sum\left\{(-1)^{|p|} D^{p}\left(a_{p q} D^{q} u\right):|p|,|q| \leqq m\right\}, \quad u \in D(A) .
$$

To determine $D(A)$, we define

$$
V=\left\{v \in H^{m}(G): B_{j} v=0 \text { on } \partial G, 0 \leqq j \leqq p-1\right\},
$$

where $0 \leqq p \leqq m$ and each $B_{j}$ is a normal boundary differential operator of (normal) order $m_{j}<m[4],[10]$. We choose additional boundary operators to augment this set and then obtain by Green's formula a collection $\left\{B_{j}: p \leqq j \leqq m\right.$ $-1\}$ of normal boundary operators with $m \leqq m_{j}<2 m, p \leqq j \leqq m-1$. (See, e.g., [10, II. 9] for details.) Assume that the partial differential operator (2.5) and the boundary operators $\left\{B_{j}: 0 \leqq j \leqq m-1\right\}$ constitute a regular boundary value problem on $G$. Then $D(A)=\left\{v \in H^{2 m}(G): B_{j} v=0\right.$ on $\left.\partial G, 0 \leqq j \leqq m-1\right\}$ and we have the estimate

$$
|A u| \geqq c_{3}\|u\|_{2 m}, \quad u \in D(A),
$$

where $c_{3}>0$.

We apply the interpolation theorem to our regular boundary value problem. Considering the identity map of $H \rightarrow H$ and $D(A) \rightarrow H^{2 m}(G)$, we obtain from the estimate (2.6) and the identity $\left[H^{j}(G), H^{k}(G)\right]_{\alpha}=H^{l}(G), l=(1-\alpha) j+\alpha k$, the estimates

$$
\left|A^{k / 2 m} u\right| \geqq c_{4}\|u\|_{k}, \quad u \in D(A), \quad 0 \leqq k \leqq 2 m .
$$

If the boundary value problem is $k$-regular, $k \geqq 1$, that is, $A u \in H^{r}(G)$ implies $u \in H^{2 m+r}(G)$ with

$$
\|A u\|_{r} \geqq c^{(k)}\|u\|_{2 m+r}, \quad 0 \leqq r \leqq k,
$$

then we obtain the estimate

$$
\left|A^{1+k / 2 m} u\right| \geqq c_{1}^{(k)}\|u\|_{2 m+k},
$$

where powers of $A$ larger than one are defined by composition or, e.g., as in $[3$, II. 14]. Finally, we recall that Grisvard [4] characterized the domains of the 
fractional powers by $D\left(A^{\theta}\right)=\left\{v \in H^{2 m \theta}(G): B_{j} v=0\right.$ on $\partial G$ if $\left.m_{j}<2 m \theta-\frac{1}{2}\right\}$ whenever $0 \leqq \theta \leqq 1$ and $2 m \theta-\frac{1}{2}$ was not an integer. (We shall be concerned only with the case in which $2 m \theta$ is an integer.)

Let $f \in H$ and consider the problem of approximating the $u \in D(A)$ for which $A u=f$. The solution is determined by the variational equality

$$
u \in V, \quad a(u, v)=(f, v), \quad v \in V,
$$

since $V$ is dense in $H$. The Galerkin approximation of $u$ determined by a given finite-dimensional subspace $M$ of $V$ is the solution $w$ of the problem

$$
w \in M, \quad a(w, v)=(f, v), \quad v \in M .
$$

Then $w$ is the projection of $u$ onto $M$ with respect to the bilinear form $a(\cdot, \cdot)$, and (2.10) is an algebraic problem for the coefficients of the expansion of $w$ by a basis for $M$. We shall assume that $M$ belongs to a family $\mathscr{M}=\left\{M_{h}: 0<h<1\right\}$ of finite-dimensional subspaces of $V$ which satisfy the following approximation assumption: there is an integer $k \geqq m$ depending on $\mathscr{M}$ and a constant $c>0$ depending on $\mathscr{M}$ and the regular boundary value problem such that the Galerkin approximation $w \in M$ of the solution $u \in H^{k}(G)$ of (2.9) satisfies the error estimate

$$
\begin{array}{ll}
|u-w| \leqq c h^{2(k-m)}\|u\|_{k} & \text { if } m \leqq k \leqq 2 m, \\
|u-w| \leqq c h^{k}\|u\|_{k} & \text { if } 2 m \leqq k .
\end{array}
$$

Such $L^{2}$-estimates are typical, e.g., for finite element spaces of degree $k-1$ with mesh parameter $h>0$ [11, Thm. 3.7].

3. Parabolic boundary value problems. Let $A$ be the unbounded operator on $H$ constructed from a continuous coercive bilinear form as in $\$ 2$. Then $-A$ generates an analytic semigroup $\{S(t): t \geqq 0\}$ of contractions on $H$, and for each $u_{0} \in H$ the function $u(t) \equiv S(t) u_{0}$ is the unique solution of (1.1). In particular, $u \in C([0, \infty), H)$ and at each $t>0, u$ is analytic with $u(t) \in D\left(A^{p}\right)$, all $p \geqq 0$, and satisfies $u^{(k)}(t)=(-A)^{k} u(t)$ for integer $k \geqq 0$. For $\beta \geqq 0$ and $t>0$ we have the estimates

$$
\left\|A^{\beta} S(t)\right\|_{L(H)} \leqq M_{\beta} / t^{\beta}
$$

(See [3, II. 14] or [7, IX. 1.6] for details.)

Suppose, hereafter, that $u_{0} \in D\left(A^{\alpha}\right)$ for some $\alpha \geqq 0$. Since each fractional power of $A$ commutes with the semigroup, the estimate above gives

$$
\left|A^{\beta} u(t)\right| \leqq\left(M_{\beta-\alpha} / t^{\beta-\alpha}\right)\left|A^{\alpha} u_{0}\right|, \quad \beta \geqq \alpha, \quad t>0 .
$$

Similarly, since each $S(t)$ is a contraction on $H$ we obtain

$$
\left|A^{\alpha} u(t)\right| \leqq\left|A^{\alpha} u_{0}\right|, \quad t \geqq 0,
$$

and $u \in C\left([0, \infty), D\left(A^{\alpha}\right)\right)$.

The solution of (1.1) satisfies the variational equation

$$
\left(u^{\prime}(t), v\right)+a(u(t), v)=0, \quad v \in V, \quad t>0 .
$$


If $M$ is a finite-dimensional subspace of $V$, we define a corresponding FaedoGalerkin approximation of $u(\cdot)$ as the solution $U \in C^{1}([0, \infty), M)$ of

$$
\left(U^{\prime}(t), v\right)+a(U(t), v)=0, \quad v \in M, \quad t>0,
$$

with $U(0)$ to be prescribed below. (Since $M$ has finite dimension, (3.4) is equivalent to a system of ordinary differential equations.)

Assume hereafter that $2 m \alpha$ is an integer and $\alpha \geqq \frac{1}{2}$. Since $D\left(A^{1 / 2}\right)=V$ ([9], [10]) we have $u \in C([0, \infty), V)$ and we can define $W \in C([0, \infty), M)$ as the pointwise elliptic Galerkin projection onto $u$ :

$$
a(W(t), v)=a(u(t), v), \quad v \in M, \quad t \geqq 0 .
$$

It follows then that we have

$$
a\left(W^{\prime}(t), v\right)=a\left(u^{\prime}(t), v\right), \quad v \in M, \quad t>0 .
$$

Following [11], we have from (3.3), (3.4) and (3.5),

$$
\left(u^{\prime}(t)-W^{\prime}(t), v\right)=\left(U^{\prime}(t)-W^{\prime}(t), v\right)+a(U(t)-W(t), v), \quad v \in M, \quad t>0,
$$

and setting $v=U(t)-W(t)$ gives

$$
\left(\frac{1}{2}\right) D_{t}\left(|U(t)-W(t)|^{2}\right) \leqq\left(u^{\prime}(t)-W^{\prime}(t), U(t)-W(t)\right) .
$$

Note that if $f$ is a continuously differentiable $H$-valued function, then it is locally Lipschitz and so then is $|f(t)|$. Hence, $|f(t)|$ is differentiable almost everywhere. Applying this remark to the above estimate gives

$$
|U(t)-W(t)| D_{t}(|U(t)-W(t)|) \leqq|U(t)-W(t)| \cdot\left|u^{\prime}(t)-W^{\prime}(t)\right|
$$

for almost every $t>0$. Consider the set $Z \equiv\{t>0:|U(t)-W(t)|=0\}$. If $t$ is not in this set, then we have

$$
D_{t}|U(t)-W(t)| \leqq\left|u^{\prime}(t)-W^{\prime}(t)\right| .
$$

If $t$ is an accumulation point of $Z$ then the indicated derivative is zero and (3.7) holds. But there are a countable number of isolated points of $Z$, so (3.7) holds almost everywhere, and we can integrate it to obtain

$$
|U(t)-W(t)| \leqq|U(0)-W(0)|+\int_{0}^{t}\left|u^{\prime}-W^{\prime}\right|, \quad t \geqq 0
$$

From the triangle inequality we obtain the fundamental error estimate

$$
|u(t)-U(t)| \leqq|u(t)-W(t)|+|U(0)-W(0)|+\int_{0}^{t}\left|u^{\prime}-W^{\prime}\right|, \quad t \geqq 0 .
$$

We can easily estimate the error contributed by the first term in (3.8). If $\frac{1}{2} \leqq \alpha \leqq 1$, we obtain from (2.11), (2.7) and (3.2),

$$
|u(t)-W(t)| \leqq \text { const. })\left|A^{\alpha} u_{0}\right| \cdot \begin{cases}h^{2 m(2 \alpha-1)}, & 2 m \alpha \leqq k \\ h^{2(k-m)}, & m \leqq k \leqq 2 m \alpha\end{cases}
$$


Similarly, if $\alpha \geqq 1$ we obtain the estimates

$$
|u(t)-W(t)| \leqq \text { const. })\left|A^{\alpha} u_{0}\right| \cdot \begin{cases}h^{2 m \alpha}, & 2 m \alpha \leqq k \\ h^{k}, & 2 m \leqq k \leqq 2 m \alpha \\ h^{2(k-m)}, & m \leqq k \leqq 2 m\end{cases}
$$

We intend to obtain from (3.6) estimates of the same order in $h$ for the third term in (3.8) on the interval $0 \leqq t \leqq T$, and we consider the preceding five cases separately.

Case 1. $\frac{1}{2}<\alpha \leqq 1,2 m \alpha<k$. Let $0<\delta<T$. Then (2.11), (1.1), (2.7) and (3.1) give

$$
\begin{aligned}
\int_{0}^{\delta}\left|u^{\prime}-W^{\prime}\right| & \leqq \text { const. }\left|A^{\alpha} u_{0}\right| \int_{0}^{\delta} t^{\alpha-3 / 2} d t \\
& =\text { const. }\left|A^{\alpha} u_{0}\right| \delta^{\alpha-1 / 2},
\end{aligned}
$$

and, similarly, we obtain (with $k \leqq 2 m$ )

$$
\begin{aligned}
\int_{\delta}^{T}\left|u^{\prime}-W^{\prime}\right| & \leqq \int_{\delta}^{T}\|A u\|_{k} \cdot h^{2(k-m)} \leqq \text { const. }\left|A^{\alpha} u_{0}\right| \int_{\delta}^{T} t^{\alpha-1-k / 2 m} d t \\
& \leqq \text { const. }\left|A^{\alpha} u_{0}\right| \delta^{\alpha-k / 2 m} \cdot h^{2(k-m)} .
\end{aligned}
$$

Adding these with $\delta=h^{4 m}$ gives

$$
\int_{0}^{T}\left|u^{\prime}-W^{\prime}\right| \leqq \text { const. }\left|A^{\alpha} u_{0}\right| \cdot h^{2 m(2 \alpha-1)} .
$$

Case 2. $\frac{1}{2}<\alpha \leqq 1, m \leqq k \leqq 2 m \alpha$. If $k=2 m \alpha$, then for each $\varepsilon>0$ we can apply the proof of Case 1 with $\alpha$ replaced by $\alpha-\varepsilon$ to obtain

$$
\int_{0}^{T}\left|u^{\prime}-W^{\prime}\right| \leqq \text { const. }\left|A^{\alpha-\varepsilon} u_{0}\right| \cdot h^{2 m(2 \alpha-1-2 \varepsilon)} .
$$

But (2.4) shows $\left|A^{\alpha-\varepsilon} u_{0}\right| \leqq c_{1}^{-\varepsilon}\left|A^{\alpha} u_{0}\right|$, so

$$
\int_{0}^{T}\left|u^{\prime}-W^{\prime}\right| \leqq \text { const. }\left|A^{\alpha} u_{0}\right| \cdot h^{2 m(2 \alpha-1-2 \varepsilon)},
$$

where the constant is independent of $\varepsilon$, and we obtain (3.9). If $k<2 m \alpha$ we obtain the estimate

$$
\begin{aligned}
\int_{0}^{T}\left|u^{\prime}-W^{\prime}\right| & \leqq C \int_{0}^{T}\|A u\|_{k} \cdot h^{2(k-m)} \\
& \leqq \text { const. }\left|A^{\alpha} u_{0}\right| \int_{0}^{T} t^{\alpha-1-k / 2 m} d t \cdot h^{2(k-m)} \\
& =\text { const. }\left|A^{\alpha} u_{0}\right| \cdot h^{2(k-m)}
\end{aligned}
$$

from (2.11), (2.7) and (3.1). 
Case 3. $1<\alpha, 2 m \alpha \leqq k$. Let $0<\delta<T$ and assume $k=2 m \alpha$. For each $\varepsilon, 0<\varepsilon<1$, we have from (2.11), (2.8), (3.1) and (2.4),

$$
\begin{aligned}
\int_{\delta}^{T}\left|u^{\prime}-W^{\prime}\right| & \leqq C \int_{\delta}^{T}\|A u\|_{k} \cdot h^{k} \leqq \text { const. } \int_{\delta}^{T}\left|A^{1+\alpha} u\right| \cdot h^{k} \\
& \leqq \text { const. }\left|A^{\alpha-\varepsilon} u_{0}\right| \int_{\delta}^{T} t^{-1-\varepsilon} d t \cdot h^{k} \\
& \leqq \text { const. }\left|A^{\alpha} u_{0}\right| \cdot \delta^{-\varepsilon} \cdot h^{k},
\end{aligned}
$$

where the constant is independent of $\varepsilon$ and $\delta$. Letting $\varepsilon \rightarrow 0^{+}$, then $\delta \rightarrow 0^{+}$, we obtain

$$
\int_{0}^{T}\left|u^{\prime}-W^{\prime}\right| \leqq \text { const. }\left|A^{\alpha} u_{0}\right| \cdot h^{2 m \alpha} .
$$

Case 4. $1<\alpha, 2 m \leqq k<2 m \alpha$. Choose $\beta$ so that $2 m \beta=1+k$. Then $\beta \leqq \alpha$, so

$$
\begin{aligned}
\int_{0}^{T}\left|u^{\prime}-W^{\prime}\right| & \leqq \text { const. } \int_{0}^{T}\left|A^{1+k / 2 m} u\right| \cdot h^{k} \\
& \leqq \text { const. }\left|A^{\beta} u_{0}\right| \int_{0}^{T} t^{-1+1 / 2 m} d t \cdot h^{k} \\
& \leqq \text { const. }\left|A^{\alpha} u_{0}\right| \cdot h^{k} .
\end{aligned}
$$

Case 5. $1<\alpha, m \leqq k \leqq 2 m$. The preceding procedure gives the estimate

$$
\int_{0}^{T}\left|u^{\prime}-W^{\prime}\right| \leqq \text { const. }\left|A^{\alpha} u_{0}\right| \cdot h^{2(k-m)} .
$$

Finally, we note that if $U(0)$ is chosen by any of the usual methods, i.e., interpolation, Galerkin projection or $L^{2}$-projection, the resulting estimates are (at least) as good as the above. The preceding discussion is summarized in the following.

THEOREM. With the notation given in $\$ 2$, let $V$ be a closed subspace of $H^{m}(G)$ determined by a (possibly empty) collection of normal homogeneous boundary operators of order $<m, a(\cdot, \cdot)$ be the given continuous and coercive bilinear form on $V$, and let $A$ be the elliptic partial differential operator (2.5) of order $2 m$ determined on $L^{2}(G)$ by a choice of additional boundary operators and Green's theorem on $G$. Let $u_{0} \in L^{2}(G)$ and denote by $u(\cdot)$ the unique solution of (1.1). Let $M$ be a finite-dimensional subspace of $V$ and denote by $U(\cdot)$ the unique solution of (3.4) for which, e.g., $U(0)$ is the $L^{2}(G)$-projection of $u_{0}$ onto $M$.

Assume the following:

(i) $\left\{A, B_{j}: 0 \leqq j \leqq m-1\right\}$ is a $k$-regular elliptic boundary value problem.

(ii) $M$ is taken from a collection of subspaces of $V$ which satisfy the approximation assumption (2.11), and

(iii) $u_{0} \in D\left(A^{\alpha}\right)$, where $2 m \alpha$ is an integer $\geqq m$.

Then we have the estimate

$$
\|u(t)-U(t)\|_{L^{2}(G)} \leqq \text { const. }\left\|A^{\alpha} u_{0}\right\|_{L^{2}(G)} \cdot h^{p},
$$

where $p=2(k-m)$ if $m \leqq k \leqq 2 m \cdot \min \{\alpha, 1\}$ and $p=k$ if $2 m \leqq k \leqq 2 m \alpha$. 
Remarks.

1. The hypothesis (i) is standard and one can consult, e.g., [10] for conditions on the coeffrcients and region $G$ which imply it. Similarly, (ii) is standard [9], and (iii) can be obtained from regularity of $u_{0}$ and boundary conditions on $A^{j} u_{0}$ for $j$ integer and $\leqq$ the integer part of $\alpha[4]$.

2. In the case $k \geqq 2 m$ and $\alpha=1$ we have convergence of order $2 m$ in $h$. Trace theory [8, VII] implies that, in general, $u^{\prime} \in L^{2}\left((0, T), D\left(A^{1 / 2}\right)\right)=L^{2}((0, T), V)$, so we cannot hope to obtain (3.10) by estimating $u^{\prime}$ with the norm of $L^{2}((0, T), D(A))$ or (equivalently) $L^{2}\left((0, T), H^{2 m}(G)\right)$.

3. The assumption that $M$ be a subspace of $V$ restricts the method to natural boundary conditions or very special domains. The construction of subspaces satisfying essential boundary conditions is almost always impossible.

\section{REFERENCES}

[1] T. Dupont, Some $L^{2}$ error estimates for parabolic Galerkin methods, Mathematical Foundations of the Finite Element Method with Applications to Partial Differential Equation, Academic Press, New York, 1972.

[2] J. Bramble And V. ThOmee, Discrete time Galerkin methods for a parabolic boundary value problem, Math. Research Center Rep. 1240, Univ. of Wisconsin, Madison, 1972.

[3] A. Friedman, Partial Differential Equations, Holt, Rinehart and Winston, New York, 1969.

[4] P. Grisvard, Caracterisation de quelques espaces d'interpolation, Arch. Rational Mech. Anal., 25 (1967), pp. 40-63.

[5] T. Kato, A generalization of the Heinz inequality, Proc. Japan Acad., 6 (1961), pp. 305-308.

[6] - Fractional powers of dissipativesoperators, J. Math. Soc. Japan, 13 (1961), pp. 246-274; 14 (1962), pp. 242-248.

[7] - Perturbation Theory for Linear Operators, Springer, Berlin, 1966.

[8] J. Lions, Equations differentielles operationnelles et problemes aux limite, Springer, Berlin, 1961.

[9] - Espaces d'interpolation et domaines de puissances fractionnaires d'operateurs, J. Math. Soc. Japan, 14 (1962), pp. 233-241.

[10] J. Lions AND E. Magenes, Problems aux limites non homogenes et applications, vol. I, Dunod, Paris, 1968.

[11] G. Strang and G. Fix, An Analysis of the Finite Element Method, Prentice-Hall, Englewood Cliffs, 1973.

[12] V. ThOmEe, Some convergence results for parabolic boundary value problems, Conference on Finite Element Methods, Math. Research Center, Univ. of Wisconsin, Madison, 1974. 